\title{
Hepatosplenomesenteric trunk; a rare variation of the celiac trunk
}

\author{
Samarawickrama MB \\ Department of Anatomy, Faculty of Medicine, Karapitiya
}

Corresponding author: Dr.M.B. Samarawickrama (samaramb@gmail.com)

\section{Introduction}

Anatomical variations of the celiac trunk are known. These variations are due to the developmental abnormalities in the ventral splanchnic arteries. This case report highlights a rare variation observed during routine dissection of an 80-year-old male cadaver.

\section{Case Report}

During the dissection of an 80-year-old male cadaver, the celiac trunk was found to branch in an unusual manner. The celiac trunk arose from the ventral surface of the abdominal aorta at the level of the $1^{\text {st }}$ lumbar vertebra. The left gastric artery arose from the anterior surface of the aorta just proximal to the celiac trunk as a separate artery (Fig $1 \& 2$ ). The trunk was about $0.5 \mathrm{~cm}$ long and it gave origin to a common trunk (for the common hepatic artery and the splenic artery) and the superior mesenteric artery. The superior mesenteric artery passed deep to the pancreas. The common trunk was about $0.5 \mathrm{~cm}$ long and divided into two main branches, the splenic artery and the common hepatic artery. Both these arteries were almost equal in diameter. The splenic artery took its normal pathway along the upper border of the pancreas to the spleen. The common hepatic artery gave three branches; the first branch originated from the superior surface of the common hepatic artery and it was a common trunk for right gastric and left hepatic artery. The gastroduodenal artery originated from the inferior aspect of the common hepatic artery and passed deep to the $1^{\text {st }}$ part of the duodenum. The next branch took the normal anatomical position of the proper hepatic artery and was larger than the other two. It passed along with the common hepatic duct and gave a smaller branch to the right lobe of the liver and the artery continued as the cystic artery (Fig 3).

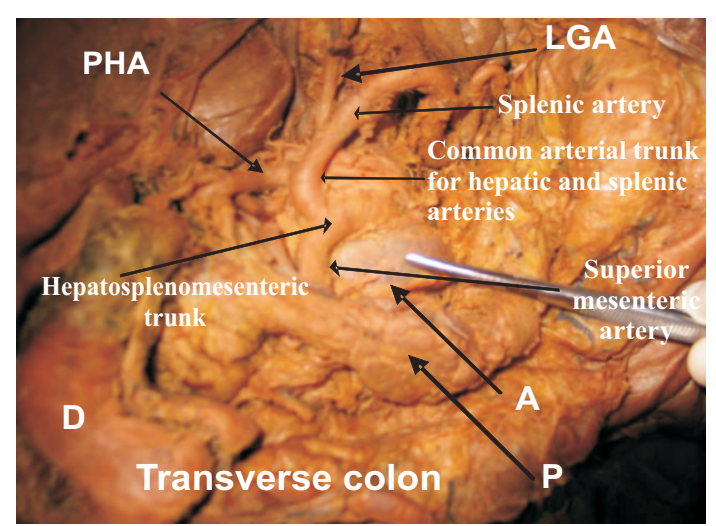

Fig 1. The stomach is raised up and pancreas is pulled down to show the area of celiac trunk. $\mathrm{A}=$ aorta, $\mathrm{P}=$ pancreas, $\mathrm{D}=$ duodenum, $\mathrm{PHA}=$ proper hepatic artery, $\mathrm{LGA}=$ left gastric artery

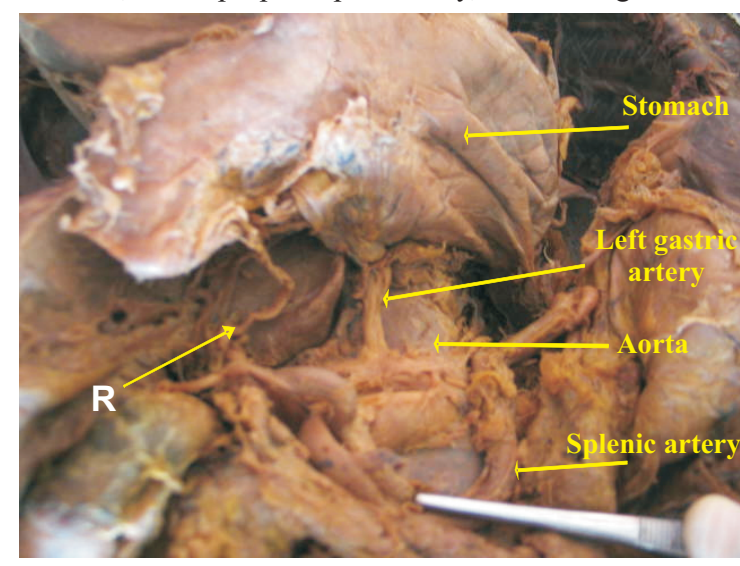

Fig 2. The stomach is separated from the duodenum and pulled up and the splenic artery is pulled down to show the left gastric artery arising directly from the aorta. $\mathrm{R}=$ right gastric artery

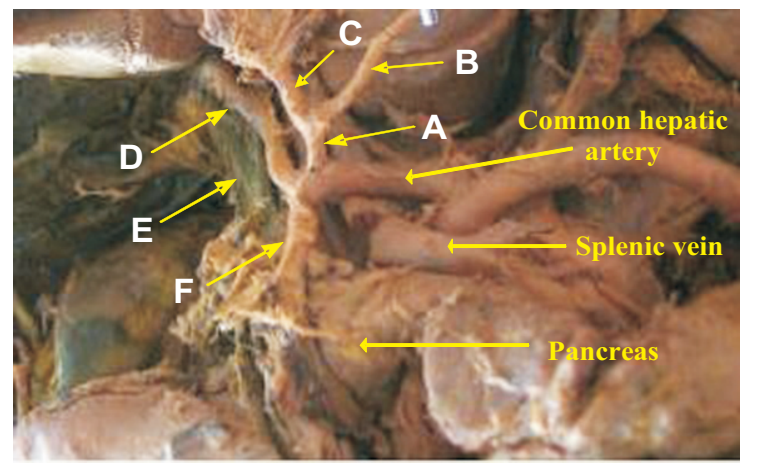

Fig 3. The stomach is separated from the duodenum and raised up while duodenum pulled down to demonstrate the branches of the common hepatic artery $\mathrm{A}=$ common trunk for right gastric and left hepatic artery, $\mathrm{B}=$ right gastric artery, $\mathrm{C}=$ left hepatic $\operatorname{artery}, \mathrm{D}=$ proper hepatic artery, $\mathrm{E}=$ common hepatic duct, $\mathrm{F}=$ gastroduodenal artery. 


\section{Discussion}

Several variations of the celiac trunk, particularly in relation to its length, diameter, location or branches (1) have been reported. Yi SQ et al reported a case of absent celiac trunk where common hepatic, splenic and gastroduodenal artery arose as separate branches of the abdominal aorta (2).

The normal anatomy of the celiac trunk is shown in the Picture 1 bellow. It is the shortest and widest ventral visceral branch of the abdominal aorta. The length ranges from 5 to $40 \mathrm{~mm}$ while the diameter range from 4 to $10 \mathrm{~mm} \mathrm{(3).} \mathrm{It} \mathrm{is} \mathrm{situated} \mathrm{between} \mathrm{the}$ T12-L1 vertebrae.

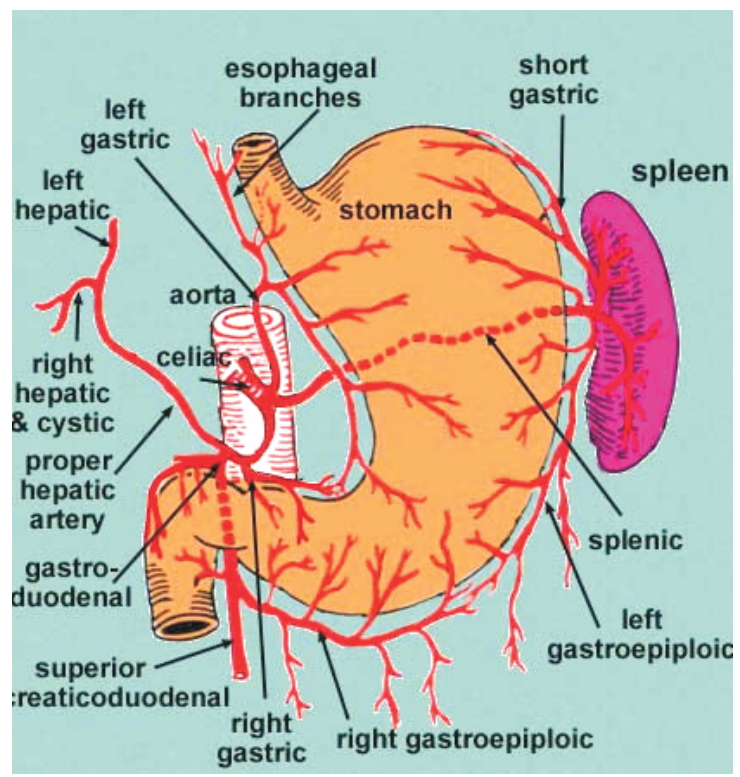

Picture 1 - The normal anatomy of the celiac trunk (home.comcast.net/ wnor/celiactrunk.jpg)

The trunk is divided into three branches and the first is the left gastric artery. The trunk gives two branches the right one is the common hepatic artery while the left one is the splenic artery. The common hepatic artery gives right gastric, gastroduodenal and the proper hepatic arteries. This typical pattern of the celiac trunk was observed only in $66.6 \%$ of the subjects in a study done on 974 cadavers (4) and 65\% in a study of 200 cadavers (5). The occurrence of the typical pattern of the hepatic arteries sited as $72.4 \%$ in the same study while Koops, A et al (6) have seen it in $79.1 \%$ of subjects.

There are six different types of variations of the celiac trunk according to the Michels classification (7, Picture 2).

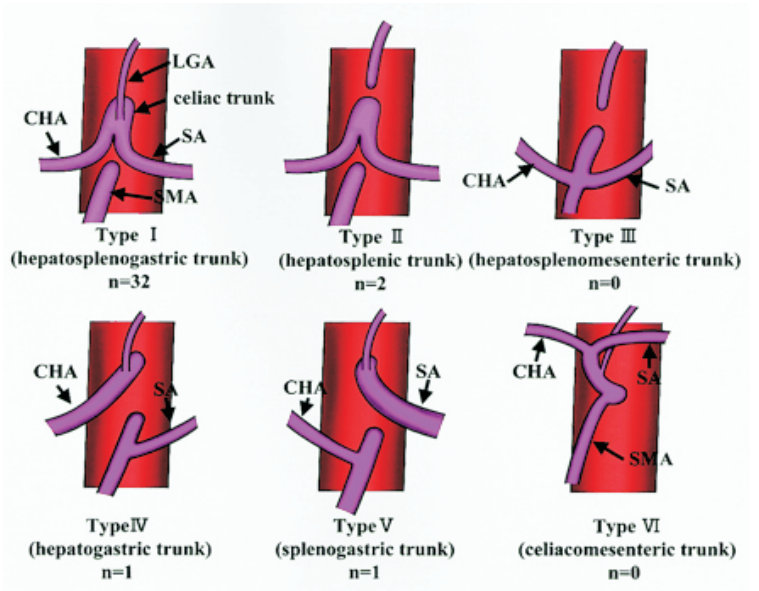

Picture 2 - Michels classification $\mathrm{CHA}=$ common hepatic artery, LGA = left gastric artery, $\mathbf{S A}=$ splenic artery, SMA = superior mesenteric artery

The independent origin of the left gastric artery from aorta, as in this case, seems to be a rare variation. This occurs in type II and III of the Michels classification. Selma Petrella et al (3) describe this in two out of 76 cadaverse $(2.25 \%)$ they studied. In those two cases the celiac trunk gave only two branches; common hepatic and the splenic artery. This type of celiac trunk is referred to as the hepatosplenic trunk (Michels type II). However, in the case described in this article the superior mesenteric artery also originated from the same arterial trunk in addition to the common hepatic and the splenic artery. This form of arterial trunk is referred to as hepatosplenomesenteric trunk and is a rarer occurrence than the hepatosplenic trunk. The rare occurrence of this variation is stated to be $0.7 \%$ (4). Mitsuru Matsuki et al (8) say that they did not encounter a case of hepatosplenomesenteric trunk even though they have seen the other types. This shows the rarity of this variation.

Anatomical variations of the celiac trunk are due to development anomalies of the ventral splanchnic arteries. The knowledge of these variations is important in operative and diagnostic procedures within the supracolic region of the abdomen.

\section{References}

1. Fatih YAZAR, asan OZAN, Ömer ÖZDOĞMUŞ, Bülent YALÇIN, Necdet. Kocabiyik. Variations of the Branches of the Celiac Trunk. Gülhane Tip Dergisi 2004; 46(2):163-5. 
2. Yi SQ, Terayama H, Naito M, Hirai S, Alimujang S, Yi N, Tanaka S, Itoh M. Absence of celiac trunk: Case report and review of the literature. Clin Anat 2008; 21(4): 283-6.

3. Selma Petrella, Celio Fernando de Sousa Rodriguez, Emerson Alexandre Sgrott, Geraldo José Medeiros Fernandes, Sergio Ricardo Marques \& José Carlos Prates. Anatomy and Variations of the Celiac Trunk. Int J. Morpho 2007; 25(2): 249-57.

4. Chen H, Yano R, Emura S, Shoumura S. Anatomic variation of the celiac trunk with special reference to hepatic artery patterns. Ann Anat 2009 Jun 6; (Epub Ahead of print).

5. Michels, N. A. Variational anatomy of the hepatic, cystic, and retroduodenal arteries. Am. Med. Assoc 1953b; 66: 2034.
6. Koops A, Wojciechowski B, Broering D, Adam G, KrupskiBerdien G. Anatomic variations of the hepatic arteries in 604 selective celiac and superior mesenteric angiographies. Surgical and Radiologic Anatomy, Volume 26, Number 3, June 2004: pp. 239-44.

7. Michels NA. Blood supply and anatomy of the upper abdominal organs with a descriptive atlas. Philadelphia, PA: Lippincott, 1995.

8. Mitsuru Matsuki, Hiroyuki Kani, Fuminari Tatsugami, Shushi Yoshikawa, Isamu Narabayashi, Sang-Woong Lee, Hisashi Shinohara, Eiji Nomura and Nobuhiko Tanigawa. Preoperative Assessment of Vascular Anatomy Around the Stomach by 3D Imaging Using MDCT Before Laparoscopy-Assisted Gastrectomy AJR 2004; 183:14551.

\title{
Retroperitoneal haemorrhage following a hump-nosed viper (Hypnale hypnale) bite; a late presentation
}

\author{
Sunanda H, Tilakaratne S, Coomaraswamy W \\ Teaching Hospital, Karapitiya, Galle \\ Corresponding author:Dr.H.Sunanda (sunanda.hediwattege@gmail.com)
}

\section{Introduction}

Hump-nosed viper bite is common in Sri Lanka, accounting for $27-35 \%$ of all snake bites $(1,2)$. Hump-nosed viper is categorized as a "non-lethal snake" but spontaneous systemic bleeding occurs in $18 \%$ patients (2). There is no effective antivenom developed against Hypnale hypnale venom. Current literature states that the hypnale coagulopathy lasts for one to three weeks (3). We report a case of coagulopathy, manifested late, in a patient with hump-nosed viper bite. Search of PubMed using MeSH terms "snake", "hemorrhage" and "viper" did not reveal any literature to support this type of late presentation.

\section{Case Report}

A, previously well, 49-year old housewife was admitted with an acute onset swelling and pain of left lower limb associated with lower abdominal pain and syncopy of one day duration. There was no history of fever and her last regular menstrual period was 11 days ago. There was a wound over her right thumb following a hump-nosed viper (Hypnale hypnale) bite five weeks back. She gave no history of a fall or trauma to the abdomen.

On admission she was in pain but not pale, febrile or dyspnoeic. Pulse rate was $120 / \mathrm{min}$ and the respiratory rate was $28 / \mathrm{min}$. Her blood pressure was $90 / 60 \mathrm{mmHg}$. The left lower limb was grossly swollen but it was not warm or had no erythema. There was no inguinal lymphadenitis. Her lower abdomen was tender and there was guarding. Per rectal examination did not reveal any contact bleeding or masses and per vaginal examination was not done because of intense pain.

Initial investigations showed haemoglobin of $9.2 \mathrm{~g} / \mathrm{dL}$, white cell count of $15.4 \times 10^{9} / \mathrm{L}$ (Neutrophils $78 \%$ ), platelet count of $200 \times 10^{9} / \mathrm{L}$, APTT of 110 seconds and INR of 1.5. Ultrasound scan revealed a round solid mass measuring $7.1 \times 5.5 \mathrm{~cm}$, situated anterior and left to the bladder. Other abdominal and pelvic organs were normal and there was a mild ascites. 